\title{
Fall Risk Reduction for the Elderly by Using Mobile Robots Based on Deep Reinforcement Learning
}

\author{
Takaaki Namba* \\ Graduate School of Engineering, Nagoya University, \\ Furo-cho, Chikusa-ku, Nagoya City, 464-8603, Japan \\ Yoji Yamada \\ Graduate School of Engineering, Nagoya University, \\ Furo-cho, Chikusa-ku, Nagoya City, 464-8603, Japan \\ E-mail: namba.takaaki@b.mbox.nagoya-u.ac.jp, । RMUI DPDOD\# PDHQDJRI DIXIDFIN \\ www.nagoya-u.ac.jp
}

\begin{abstract}
Slip-induced fall is one of the main factors causing serious fracture injuries among the elderly. In this paper, we propose a fall risk reduction measures for the elderly, based on deep reinforcement learning using mobile assistant robots. Our method involves online real-time risk analysis and risk reduction. The results suggest that our method is applicable to the prevention of not only slip-induced fall, but also other factors related to falling and other cases of accidents.
\end{abstract}

Keywords: Safety, risk reduction, mobile robot, deep learning, reinforcement learning.

\section{Introduction}

In some countries, including Japan, there is an increasing tendency in the number of accidents involving the elderly. Slip-induced falls are one of the main factors causing serious fracture injuries among the elderly, subsequently leading to them being bed ridden. To reduce the increasing fall accidents among the elderly, we propose an approach using mobile assistant robots. We consider safety in environments wherein humans and assist robots coexist to enhance the fields of medical welfare and assist living ${ }^{1-3}$.

This paper proposes deep reinforcement learning (hereinafter called 'DQN')-based fall risk reduction measures by employing mobile assistant robots for the elderly. First, we collect preparatory data regarding past

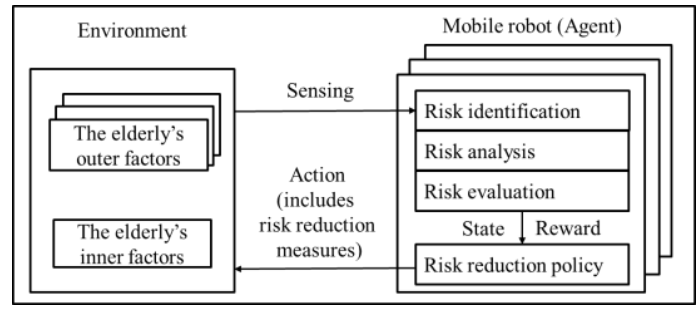

Fig. 1. Reinforcement learning for fall risk reduction of the elderly.

incidents and accidents as the input data to analyze fall risks, and to assess the examples of risk reduction measures. Second, we use a deep convolutional neural network $^{4}$ to analyze the fall risks of the elderly. Third, we apply DQN ${ }^{5-6}$ to control mobile robots according to the

*Panasonic Advanced Technology Development Co., Ltd, Nagoya Mitsui Building New Building 11F, 1-24-20 Meieki-minami, Nakamura-ku, Nagoya City, 450-0003, Japan. namba.takaaki@jp.panasonic.com 
results of risk analysis, and consequently reduce the slipinduced fall risks of the elderly.

\section{Data Preparation}

We collect the images and movies, including information of the location where the real incidents and accidents had occurred, and where similar hazards exist. In addition to these data, we use open datasets, such as Caltech101 datasets. Moreover, we expand the data by 14 times using data augmentation such as additional noise, rotation, zoom-in, zoom-out, foreground, and background. We set 128 labels as risk levels ${ }^{3}$ based on the risk classification of the international standard ISO12100 for all collected data.

\section{Method for risk analysis}

Risk analysis is carried out through methods such as Fault Tree Analysis (FTA), ${ }^{7}$ Hazard and Operability Study (HAZOP), ${ }^{8-9}$ and Failure Modes Effects and Criticality Analysis (FMECA). ${ }^{10}$ Conventional risk analyses conducted manually or partially use a probability calculation tool when employed in applications such as system safety requirement analysis or design. This type of analysis has the following limitations:

- Failure in identifying that hazards may occur owing to differences in the proficiency of the safety management officers, that is, their experience and capability,

- $\quad$ Risk analysis procedures are complex and require a specific number of man-hours depending on the scale of the assessment.

Most analyses and assessments are carried out offline based on prior information, and require time to be conducted; the results are not immediately available. Therefore, the measures of risk reduction are often delayed, and it becomes difficult to respond promptly and flexibly in continually changing situations.

We develop an online real-time risk analysis based on the deep convolutional neural network. The input is environmental sensing data aimed at detecting Hazard Elements (HEs), Initiating Mechanisms (IMs, triggers of the accident), and Target and Threat (T/T) with reference to Ericson's Hazard Theory ${ }^{11}$. According to this theory, an accident (mishap) occurs when HE, IMs, and T/T appear simultaneously at a specific time. In our study, the sensing data are mainly time-series images of the environment and distance information to the objects and parts of human body.
Table 1. Definitions of designed parameters for deep reinforcement learning.

\begin{tabular}{|l|l|}
\hline $\begin{array}{l}\text { Designed } \\
\text { parameter }\end{array}$ & Components \\
\hline Action & $\begin{array}{l}34 \text { actions (32 directions (including avoid hazard) }+ \\
1 \text { stop }+1 \text { remove hazard). }\end{array}$ \\
\hline State & $\begin{array}{l}\text { Current sensing data by a multi-spectrum camera on } \\
\text { the agent (e.g., the distance to the object by a stereo } \\
\text { camera and by a laser range finder), } \\
\text { current position and posture of the agent, } \\
\text { current goal position (toilet or hospital room), } \\
\text { time (current step count), and previous action. }\end{array}$ \\
\hline Reward & $\begin{array}{l}\text { Reachability (reach goal), transfer efficiency } \\
\text { (approach the goal; minimum steps, timeout, and } \\
\text { inertia), and safety (change in the risk: remove } \\
\text { hazard, avoid hazard). }\end{array}$ \\
\hline Experiment & $\begin{array}{l}\text { Previous state, previous action, current reward, and } \\
\text { current state. }\end{array}$ \\
\hline
\end{tabular}

The main output is the result of risk analysis and the level of risk as quantitative information. The risk is presented using the following formula:

$$
\begin{aligned}
& \text { Risk }=R(S, P h) . \\
& P h=P(F, P s, A) .
\end{aligned}
$$

Eq. (1) shows that the risk is a product of the severity of the harm $(S)$ and the probability of the harm $(P h)$. This is one of the principal criterion of ISO $12100^{12}$. Eq. (2) indicates that the probability of the harm $(P h)$ is a product of the following: the frequency of exposure to hazards $(F)$, probability of occurrence of hazardous event $(P s)$, and possibility of avoiding or limiting the harm $(A)$. We classify the risk level in 128 categories. Furthermore, according to the result of risk analysis, we generate an image of the future by blending a representative hazardous image per risk category with the current image.

\section{Method for risk reduction}

In general, regarding the safety of machinery, risk reduction is implemented by using methods such as inherently safe design measures, safeguarding implementation of complementary protective devices, and information for use. ${ }^{12-14}$ In addition, risk reduction can be performed by learning from previous incidents and accidents $^{15}$, and this can further improve the environment; for example, removing hazardous elements, avoiding hazardous situations, and ceasing the triggering of incidents and accidents. 
In this paper, we propose online real-time risk reduction measures based on DQN. Figure 1 portrays our concept. The inputs are the environmental data involving the elderly individual's outer and inner factors. The risk of the outer factors is estimated by a variety of sensing data, for example, images and distance information obtained from a stereo camera and a laser range finder. The risk of the inner factors is estimated by the elderly individual's physical condition; for example, changes in the breathing obtained from radio sensors on the mobile robots, and stability measures calculated from the signals of the accelerometers mounted in the individuals. Another input is the risk level, which is the result of risk analysis as one of the received rewards. The risk reduction policy selects an action using a deep neural network. The output is one of the actions including the risk reduction measures.

Table 1 lists the definitions of the parameters that must be determined by humans for DQN. Each component of the parameters is normalized from 0 to \pm 1.00. This DQN is trained with the Q-learning algorithm, ${ }^{5-6}$ by the epsilon-greedy strategy, and uses Adam ${ }^{16}$ for the parameter optimization. To accelerate the convergence rate of parameters, we apply experience replay, and reduce epsilon according to the learning status. Furthermore, to avoid over-fitting, we apply early stopping, use the generated model for our other scenes, and use dropout in the neural network.

\section{Experiments}

We performed experiments using a computer simulation. Figure 2 illustrates the conditions of the environment and agents. Mainly four types of scenes are shuffled and experimented upon. The hospital room and the toilet are located at random within a certain range. As a DQNbased agent, the red circle " $\mathrm{R}$ " is an autonomous mobile robot that assists the elderly. The scenario is that agent "R" goes from the hospital room to the toilet at a certain interval, in the hospital. The floor is partially wet around the toilet at random spatiotemporally, and agent "R" detects those area. The fan-shaped area shows the sensing range of agent "R". Agent " $R$ " assists the prevention of slip-induced falls for the elderly. Moreover, we compare the DQN-based agent with conventional rule-based agent according to the potential method.

\section{Results and discussion}

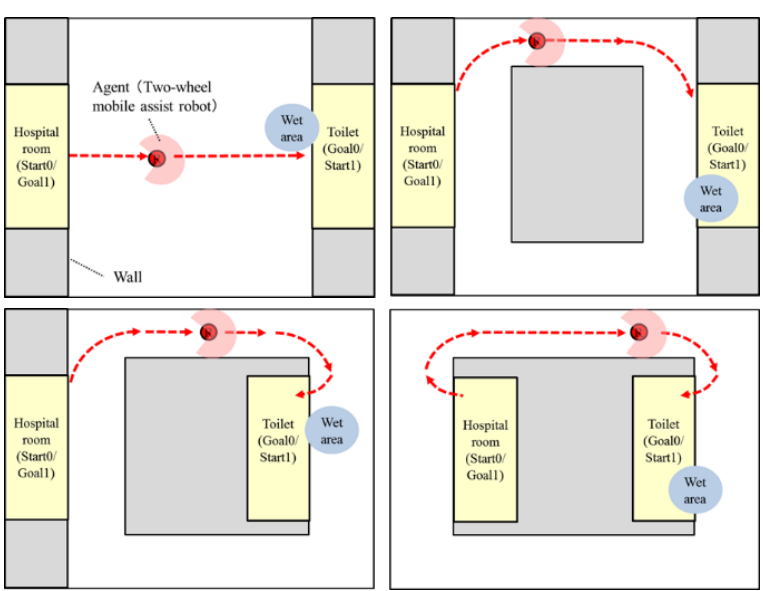

Fig. 2 Simulation environment for experiments.

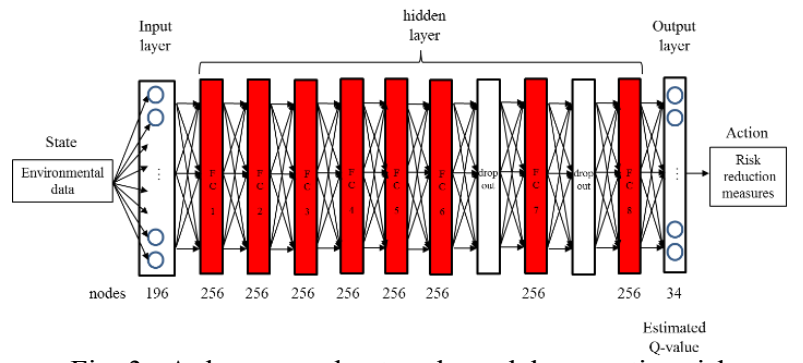

Fig. 3 A deep neural network model generating risk reduction measures.

Figure 3 depicts a feasible example of the deep neural network model that estimates the Q-value, and decides the risk reduction measures. We apply a deep fully connected network with nine layers. The deep convolutional neural network is not necessarily required to be applied to the scenes depicted in Figure 2, because we can determine the features of the environment by the combination of the components of the input data, without dimension reduction. The data of the input layer composes a normalized state vector. A state parameter includes the sensing data, robot's position, posture, goal position, and time information as environmental data. The data of the output layer shows each estimated Qvalue for actions. Each activation function is a leaky ReLU. The initial value of epsilon $\varepsilon$, which indicates the probability of random action, is set as 0.2 . The reduction rate of epsilon is 0.00005 . The discount rate of the future reward is $\gamma=0.9$.

Figure 4 describes the process explaining how the reward changes during training on the simulation. An increase in the reward indicates well-designed rewards and risk reduction. We validate the effects of our 


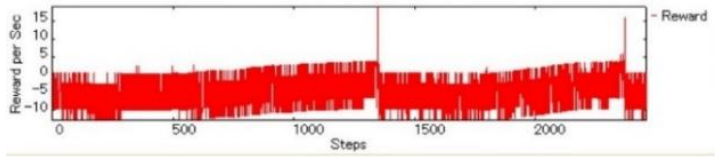

Fig. 4. Change in reward (including risk reduction) during training on the simulation. Two peaks indicate the rewards when an agent reaches the toilet or hospital room, in other words, achieves goals in two episodes.

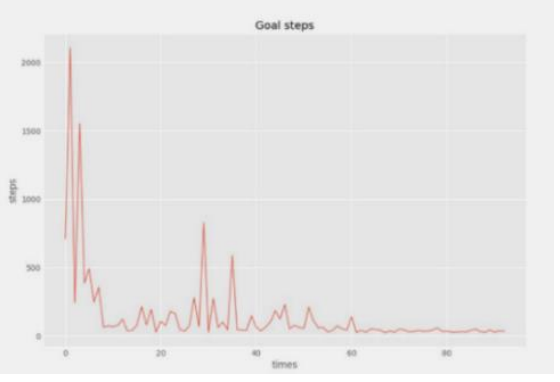

Fig. 5. Relationship between episodes and transfer efficiency as a learning curve.

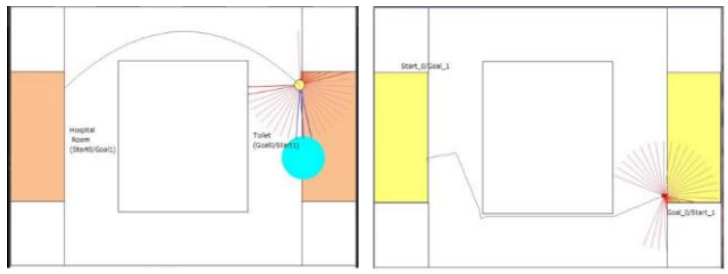

(a)

(b)

Fig. 6. Trajectories of rule-based (a) and DQN-based (b) agents

approach through the variations in the reward graph and convergence of the loss function. We noted that the deep reinforcement learning-based agent not only surpasses the rule-based agent but also automatically reduces slipinduced fall risks.

Figure 5 shows the relationship between the number of goal steps of an agent and the number of episodes. It indicates enhanced convergence of steps and stability of actions by the reinforcement learning.

Figure 6 discloses the result of comparison of the two methods. The agent in Figure 6(a) took 489 steps with the potential method, whereas the agent in Figure 6(b) took 113 steps with DQN to reach the goal. The results indicate that the transfer efficiency of the DQN agent is equal or better than that of the rule-based agent.

Furthermore, the highest priority of risk reduction measures is to "remove hazard," followed in order by "avoid hazard", and "post a warning." We verified that these policy decisions were able to control actions by adjusting the safety reward design, such as assigning a more positive reward to the action with higher priority.

Finally, the input data of our deep risk reduction network suggests that the features of our model include the local map, global map, trajectory planning, and screening for risk assessment.

\section{Conclusion}

In this paper, we propose fall risk-reduction measures for the elderly, by using mobile robots based on deep reinforcement learning, and present its usability. The results suggest that our method is applicable to not only fall prevention, but also to other factors influencing fall and other cases of accidents.

\section{References}

1. T. Namba, and Y. Yamada, A study on an assistance by mobile robots in preventing the elderly from falling, in Proc. Mechanical Engineering Congress, 2015 Japan (MECJ-15), J1630205, (in Japanese).

2. T. Namba, and Y. Yamada, A study on an assistance by mobile robots in preventing the elderly from falling (Deep Convolutional Neural Network for Fall Risk Analysis), in Proc. Mechanical Engineering Congress, 2016 Japan (MECJ-16), J1640304, (in Japanese).

3. T. Namba, and Y. Yamada, Risk Analysis Method Preventing the Elderly from Falling, in Proc. 22nd Robotics Symposia, 3A1, (2017) (in Japanese)

4. A. Krizhevsky, I. Sutskever, and G. Hinton. Imagenet classification with deep convolutional neural networks, In Advances in Neural Information Processing Systems 25, (2012), pp.1106-1114.

5. C. J. Watkins and P. Dayan, Q-learning, In Machine Learning, vol. 8, (1992), pp. 279-292.

6. V. Mnih et al. Playing Atari with Deep Reinforcement Learning". In:CoRR abs/1312.5602,(2013).

7. C. Ericson, Fault tree analysis - a history, in:17th Int System Safety Conference. Unionville, VA, (1999), pp. 1-9.

8. F. I Khan, and S. A Abbasi, Techniques and methodologies for risk analysis in chemical process industries, J. Loss Prevention in the Process Industries, Vol.11, Issue 4, (July 1998), pp. 261-277

9. J. Dunjóa, V. Fthenakis, J. A. Vílchez, and J. Arnaldos, Hazard and operability (HAZOP) analysis, A literature review, J. Hazardous Materials, Vol. 173, Issues 1-3, (15 January 2010), pp. 19-32

10. MIL-STD-1629A, Military Standard, procedures for performing a Failure Mode, Effects and Criticality Analysis, (Department of Defense, Washington, DC, 1980).

11. C. A. Ericson II, Hazard Analysis Techniques for System Safety, Second Edition, (John Wiley \& Sons, Inc., 2016).

12. ISO12100, Safety of machinery - General principles for 
design - Risk assessment and risk reduction, (2010).

13. IEC61508-ed2, Functional safety of electrical /electronic / programmable electronic safety-related systems, 2010-04.

14. ISO13482, Robots and robotic devices -- Safety requirements for personal care robots, (2014).

15. D. L. Cooke, Learning from Incidents, System Dynamics Society, (2013)

16. D. P. Kingma and J. L. Ba., Adam: A Method for Stochastic Optimization, 3rd Int. Conf. for Learning Representations (ICLR), San Diego, (2015) 